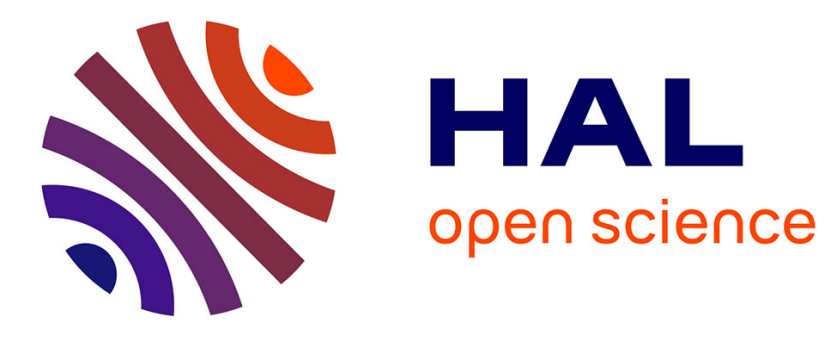

\title{
Divergences et convergences économiques
}

Françoise Berger

\section{To cite this version:}

Françoise Berger. Divergences et convergences économiques. Ulrich Pfeil, Jean-Paul Cahn (dir.). Allemagne 1961-1974. De la construction du Mur à l'Ostpolitik, éditions du septentrion, pp.187-215, 2009. halshs-00373405

\section{HAL Id: halshs-00373405 https://shs.hal.science/halshs-00373405}

Submitted on 19 May 2010

HAL is a multi-disciplinary open access archive for the deposit and dissemination of scientific research documents, whether they are published or not. The documents may come from teaching and research institutions in France or abroad, or from public or private research centers.
L'archive ouverte pluridisciplinaire HAL, est destinée au dépôt et à la diffusion de documents scientifiques de niveau recherche, publiés ou non, émanant des établissements d'enseignement et de recherche français ou étrangers, des laboratoires publics ou privés. 


\section{DIVERGENCES ET CONVERGENCES ÉCONOMIQUES}

in Ulrich Pfeil, Jean-Paul Cahn (dir.), Allemagne 1961-1974. De la construction du Mur à l'Ostpolitik, volume 2/3, éditions du Septentrion, 2009, p. 187-215.

Françoise BERGER (Grenoble)

Malgré des différences de structures assez importantes, les deux parties de l'Allemagne représentaient une des économies les plus modernes du monde industrialisé au moment de la Seconde guerre mondiale, avec un niveau de développement économique proche dans les deux ensembles ${ }^{1}$. Ces caractères n'ont pas disparu malgré la séparation en deux zones distinctes d'occupation (occidentale et soviétique) $)^{2}$, puis en deux États placés sous des régimes politiques et économiques totalement opposés. Ils ont gardé tout deux, lors de la première période de leur fonctionnement (1949-1960), des proximités tant dans la structure de la production et du capital que dans la composition de leur commerce extérieur ${ }^{3}$.

Quelques différences peuvent bien sûr être relevées, tant dans la taille globale de la population et du territoire que dans la densité de la population (plus faible à l'Est), mais aussi dans des ressources moindres, en particulier dans le domaine de l'énergie, après la perte du charbon de Silésie qui était l'approvisionnement naturel de l'industrie de l'Allemagne orientale, laquelle a dû trouver de nouvelles sources d'approvisionnement. Du fait de son incorporation dans le bloc communiste et dans un nouvel ensemble de liens économiques, la RDA a donc dû totalement redéfinir le cadre traditionnel de son commerce, ce qui a sans doute été plus long que pour la RFA et représente une rupture plus importante ${ }^{4}$.

Par ailleurs, la différence de traitement, entre les zones d'occupation puis entre les deux États, dans le domaine des destructions de guerre puis des réparations (en particulier démontages et livraisons) est bien connue. Ces deux éléments combinés ont privé la RFA de $25 \%$ de son potentiel industriel alors que la RDA a été amputée de $50 \%$ du sien. De plus, la RFA a bénéficié de 16 milliards de marks des crédits Marshall ; cependant elle a dû verser, au titre

\footnotetext{
${ }^{1}$ Le ratio de la production industrielle par habitant était même un peu supérieur à l'Est $(+16 \%)$; voir Paul Gregory, Gert LEPTIN, «Similar societies under differing economic systems : the case of the two Germanys », in : Soviet studies, 29 (oct. 1977) 4, pp. 519-542, ici p. 520.

2 À partir de la formation de la trizone (juin 1948) par regroupement d'abord des zones d'occupation américaines et britannique (bizone, en décembre 1946) puis de l'apport de la zone française.

${ }^{3}$ Idem.

${ }^{4}$ GREGORY, LEPTIN (note 1), p. 521.
} 
des remboursements du «Wiedergutmachung $»^{5}, 33$ milliards de marks, ce que la RDA n'a pas eu à faire 6 . Les deux nouveaux États ne sont donc pas entrés dans la période de l'après-guerre dans les mêmes conditions.

La période de 1961 à 1974 représente dans une certaine mesure l'apogée de la forte croissance économique de chacune des deux économies. Si ces deux États sont assez caractéristiques du système qu'ils représentent (économie de marché versus économie planifiée), et s'ils sont chacun les leaders de chaque système, ils ont cependant des spécificités fortes.

\section{Deux systèmes économiques opposés}

En 1949, la constitution des deux pays fixe des objectifs, des cadres et des méthodes différents de politique économique, de façon très précise pour la RDA, plus souple pour la RFA ${ }^{7}$. Mais ces politiques économiques ont déjà été largement orientées et fixées par les Occupants, les anglo-saxons libéraux à l'Ouest, les Soviétiques socialistes à l'Est, dès 1945.

\section{L'économie sociale de marché à l'Ouest}

Après la débâcle nazie, certains souhaitaient un démantèlement au moins partiel de l'industrie allemande et des plans ${ }^{8}$ ont circulé en 1945. Pourtant, après le contrôle total de la partie occidentale de l'Allemagne vaincue par les trois Alliés de l'Ouest, pour diverses raisons parmi lesquelles l'évidence de la guerre froide, les démontages annoncés ne furent menés au bout que très partiellement et ils n'affaiblirent donc pas durablement le potentiel économique de la future RFA, contrairement à ce qui se passa en zone d'occupation soviétique. Si certains démontages se poursuivirent jusqu'en 1949, en particulier dans la zone française, le Plan Marshall de 1948 constitue un premier changement majeur de la stratégie économique alliée vis-à-vis de

\footnotetext{
${ }^{5}$ Indemnisation. Sur ce problème, voir Walter SCHWARZ (éd.), Die Wiedergutmachung nationalsozialistischen Unrechts durch die Bundesrepublik Deutschland, München 19741986 ; Constantin. Goschler, Schuld und Schulden. Die Politik der Wiedergutmachung für NS-Verfolgte seit 1945, Göttingen, 2005.

${ }^{6}$ GREGORY, LEPTIN (note 1), p. 521.

${ }^{7}$ Eckard JeSSE (éd.), Bundesrepublik Deutschland und Deutsche Demokratische Republik. Die beiden deutschen Staaten im Vergleich, Berlin, 1982, p. 349.

${ }^{8}$ Cf. pour le Plan Morgenthau : Lothar KeTTENACKER, « Der Morgenthau-Plan. Geschichte einer Totgeburt », in : Gerhard A. RITTER (éd.), Rivalität und Partnerschaft. Studien zu den deutsch-britischen Beziehungen im 19. und 20. Jahrhundert. Festschrift für Anthony J. Nicholls, Paderborn, 1999, pp. 249-265.
} 
l'Allemagne de 1'Ouest ${ }^{9}$. Ajoutée aux autres aides directes ou indirectes, l'ensemble de l'aide américaine et britannique atteignit quatre milliards de dollars $^{10}$. Même si cet apport est loin de constituer la seule explication du redémarrage rapide de l'économie allemande et de sa croissance exceptionnelle dans les années qui suivirent, cette décision d'aider l'Allemagne a été la première étape de son intégration dans 1'Union européenne des paiements (UEP) puis dans l'organisation économique de coopération européenne (OECE), tout en contribuant à alléger la dette des réparations et à soutenir le commerce extérieur à ses débuts ${ }^{11}$. Dans le domaine du commerce extérieur, la zone d'occupation occidentale ${ }^{12}$ n'avait pas le choix de sa stratégie, elle était sous le contrôle des autorités d'occupation $^{13}$. Ainsi, l'Allemagne de l'Ouest devait entrer dans le système libéral, multilatéral du commerce mondial et les contraintes de son retour dans l'économie mondiale avaient été fixées par les Américains ${ }^{14}$.

L'Allemagne avait été, depuis le dernier quart du XIX ${ }^{\mathrm{e}}$ siècle, le pays par excellence de l'intervention de l'État dans la sphère économique et sociale, et plus encore lors de la Grande crise à la fin des années 1920 qui favorisa la prise du pouvoir par Hitler. Or après 1945, sous l'influence américaine, mais aussi sous celle de la doctrine de l'ordolibéralisme (ou néolibéralisme allemand, sous l'influence de l'École de Fribourg et de Wilhelm Röpke) ${ }^{15}$, l'Allemagne se convertit totalement au libéralisme économique. Selon le concept de l'ordolibéralisme, l'ordre économique doit aussi être moral, c'està-dire respecter les aspirations à la justice et à l'égalité, mais aussi les désirs matériels de l'homme. Il faut cependant éviter les empiètements abusifs de l'administration par la création d'un État de droit auquel le régime économique mis en place correspond. Ainsi, l'action de l'État sur le processus économique doit rester la plus limitée possible et laisser une place première à la libre initiative des individus responsables. Ceci implique la mise en place de mesures régulatrices dont les plus importantes portent sur la lutte contre les monopoles et ententes (Office des cartels), mais aussi un contrôle de la politique sociale des entreprises et un principe de redistribution du pouvoir

\footnotetext{
${ }^{9}$ Le second changement notable étant la conséquence de la Guerre de Corée ; il permit aux Allemands de l'Ouest de mettre une fin très rapide aux limitations de la production qui pesaient encore sur l'industrie en 1950.

${ }^{10}$ Gérard SCHNEILIN, Horst SCHUMACHER, Économie de l'Allemagne depuis 1945, Paris, 1992, p.21.

${ }^{11}$ Idem.

${ }^{12} \mathrm{D}$ 'abord trois zones, puis bizone et trizone (cf. note 2)

${ }^{13}$ Déc. 1947, Lovett-Strang-agreement : accord révisé pour la bizone (en fait, dicté par les Américains).

${ }^{14}$ Cf. Werner ABELSHAUSER, Deutsche Wirtschaftsgeschichte seit 1945, Bonn, 2005, pp. $217,219$.

${ }^{15}$ Cf. SCHNEILIN, SCHUMACHER (note 10), pp. 26-27.
} 
d'achat. L'ordolibéralisme allemand a donc introduit une fonction régulatrice de l'État dans l'économie qui ne soit pas incompatible avec les fondements du libéralisme, un certain équilibre entre liberté et dirigisme ${ }^{16}$.

Ainsi, l'économie de la République fédérale d'Allemagne est basée sur le modèle du marché, de la libre concurrence et de l'initiative privée des entrepreneurs. L'offre est régulée par la demande des consommateurs, et les salaires et bénéfices dépendent des mécanismes propres de régulation du marché. L'État n'intervient ponctuellement qu'en cas de crise, par des mesures de politique économique ou sociale (actions de la Bundesbank, politique conjoncturelle) $)^{17}$.

La politique "sociale de marché », une troisième voie entre libéralisme et socialisme, a été mise en place sous la conduite de Ludwig Erhard ${ }^{18}$, entre autre sous l'influence d'Alfred Müller-Armack, son proche collaborateur, créateur de cette appellation et défenseur de la «nécessaire complémentarité entre liberté, justice et égalité ${ }^{19}$. Par ailleurs, des articles de la Loi fondamentale de 1949 fixent les nécessités de rééquilibrage entre Länder riches et pauvres (investissements, etc.), tout comme la répartition des tâches financières entre Bund et Länder (et Kreis et communes). Pour autant, la Loi fondamentale ne garantit ni la neutralité de la politique économique du gouvernement, ni une économie sociale de marché mise en œuvre par les seuls moyens conformes au marché (arrêt du 20 juillet 1954), elle n'est donc pas liée au système ordolibéral, tout en contenant un certain nombre d'articles protégeant les libertés économiques fondamentales ${ }^{20}$. Ce sont finalement les succès de ce système qui vont l'ancrer définitivement, tant dans le programme de la CDU (Düsseldorf, 1949) que du SPD (Bad Godesberg, 1959).

Si l'État peut jouer sur la politique conjoncturelle, l'indépendance de la Bundesbank est essentielle dans le système allemand d'économie de marché qui fonctionne sur le crédit ${ }^{21}$. L'État n'a qu'un pouvoir monétaire restreint. Par contre, son pouvoir essentiel de contrôle se situe dans le domaine de la concurrence. Pour respecter les règles de celle-ci, une loi contre les restrictions de la concurrence a été - difficilement - mise en place (1957, suivi de la création du Bundeskartellamt) qui donne également des pouvoirs de contrôle aux ministères de 1'Économie des Länder. Cette législation est un élément fondamental du système ordolibéral ${ }^{22}$. L'État doit garantir une concurrence

\footnotetext{
${ }^{16}$ SCHNEILIN, SCHUMACHER (note 10), pp. 28-29.

${ }^{17}$ Cf. Wolfgang BeHR, Bundesrepublik Deutschland - Deutsche Demokratische Republik. Systemvergleich Politik - Wirtschaft - Gesellschaft, Stuttgart, 1985, p. 97.

${ }^{18}$ Cf. Heiner TIMMERMANN (éd.), Wirtschaftsordnungen im Dialog - Bundesrepublik Deutschland und DDR, Sarrebruck, 1989, p. 11.

${ }^{19}$ Cf. SCHNEILIN, SCHUMACHER (note 10), p. 27.

${ }^{20}$ Cf. SCHNEILIN, SCHUMACHER (note 10), p. 30.

${ }^{21}$ Cf. TIMMERMANN (note 18), pp. 14-15.

${ }^{22}$ Cf. JESSE (note 7), p. 351.
} 
non faussée: on retrouve cette idée dans les axes de la construction économique européenne, à travers la législation anticartel. Les ententes, horizontales ou verticales, sont interdites et l'Office sanctionne les abus de concentration.

En effet, la concentration des entreprises a été en progression constante autour de grosses unités : en 1966, 73,9\% des biens de production étaient déjà entre les mains de $1,7 \%$ de propriétaires. Cette concentration industrielle a eu un rôle important dans la croissance économique, tant d'ailleurs en RFA qu'en RDA $^{23}$. Pourtant, en raison des nouvelles règles du jeu économique encadré, la reconstitution de la puissance économique industrielle allemande n'est pas un simple retour à la situation d'avant-guerre (grands Konzerne ${ }^{24}$ ). Dans la plupart des cas (par exemple, le groupe Flick), on est passé du Konzern traditionnel (totale intégration verticale amont-aval) à une diversification multisectorielle ${ }^{25}$.

\section{L'économie socialiste planifiée à l'Est}

À la fin de la guerre, une grande partie de la zone orientale de l'Allemagne est détruite. C'est ce que vivent aussi les régions industrielles et les grandes villes de la partie occidentale, mais à la différence près que le poids de l'occupation soviétique s'avère bien plus dur. Si dans les deux parties de l'Allemagne, il y eut le paiement de réparations et des démontages, les livraisons se poursuivirent à l'Est jusqu'en 1953 et leur montant total dépassa largement les sommes fixées par la Commission des réparations (près de 16 milliards de dollars au lieu de 10 milliards $^{26}$ ). En particulier, les Soviétiques furent intraitables sur les démontages dans leur zone d'occupation, qui touchèrent tous les secteurs de l'économie ; ils opérèrent des confiscations d'entreprises que la RDA dut racheter par la suite et orientèrent l'économie est-allemande vers la production de biens d'investissement au détriment des biens de consommation. Ainsi, pendant les dix premières années, la politique économique de la RDA fut en grande partie alignée sur les exigences

23 Cf. Jacques Houssiaux, W. Dietrich StumMP, « Croissance, concentration et concurrence. Quelques aperçus sur l'évolution du pouvoir économique en Allemagne fédérale », in : Revue économique, (mai 1962), p. 442.

${ }^{24} \mathrm{Ce}$ terme spécifiquement allemand désigne une grande entreprise intégrée (intégration verticale le plus souvent, mais aussi horizontale). Avant la guerre, les principaux secteurs de l'industrie sont dominés par de tels Konzerne (ex. Vereinigte Stahlwerke qui produit plus de 50 de la production allemande d'acier).

${ }^{25} \mathrm{Idem}$.

${ }^{26}$ Cf. SCHNEILIN, SCHUMACHER (note 10), p. 20. 
soviétiques $^{27}$. La perte de potentiel de la zone soviétique d'occupation par rapport au niveau de 1938 a été estimée à près de $45 \%{ }^{28}$.

Outre ces prélèvements lourds, les Allemands de l'Est ne bénéficièrent pas d'une quelconque aide économique pour réparer les dégâts de guerre et investir pour le futur, comme ce fut le cas à l'Ouest. D'où, dès la création des deux États, un différentiel important dans le rythme du redressement économique de l'après-guerre et un niveau de vie qui resta, à l'Est, constamment derrière celui de l'Ouest ${ }^{29}$. De ce fait, mais aussi pour des raisons politiques, le nombre d'émigrants augmenta d'année en année. Cette menace pour l'économie dans son ensemble fut prise en compte dès 1958/59.

Un autre handicap important fut la rupture du tissu industriel de l'Allemagne orientale, qui était, avant la guerre, étroitement imbriquée avec les régions occidentales pour ses approvisionnements en énergie et en matières premières et dont la structure était plutôt orientée vers la production de biens de consommation variés. Il fallut donc réorienter les flux et construire les industries manquantes, en particulier l'industrie lourde qui s'appuya alors sur l'utilisation massive du lignite et l'importation de pétrole et de gaz soviétiques et de houille polonaise $\mathrm{s}^{30}$.

La tâche économique principale, fixée officiellement en 1953 («Neuer Kurs »), fut de remettre l'offre de biens de consommation par personne au niveau de celle de l'Allemagne de l'Ouest, alors qu'elle se situait entre $35 \%$ et $40 \%$ de celle-ci. La solution de ce problème devait passer par de nouvelles méthodes et devait être rendue possible par le renforcement de la « concurrence socialiste» dans toutes les branches industrielles ${ }^{31}$. Cependant, la priorité réelle fut de fait plutôt maintenue en faveur de l'industrie lourde, même si la production de certaines unités et certains investissements furent effectivement réorientés vers l'industrie de biens de consommation ${ }^{32}$.

En Europe, c'était la première fois qu'une économie développée entrait dans un système sur le modèle soviétique ${ }^{33}$. Alors que pour la RFA, le système économique choisi n'est pas fixé par la Loi fondamentale (sauf en ce qui concerne la liberté), en RDA, il est clairement défini par l'article 9 de la constitution $^{34}$, mais il faut cependant attendre 1967 pour que la Constitution,

${ }^{27}$ Cf. Deutsche Institut für Wirtschaftsforschung (DIW), DDR-Wirtschaft, Hamburg, 1971, pp. 56-57.

${ }^{28}$ Cf. SCHNEILIN, SCHUMACHER (note 10), p. 20.

${ }^{29}$ Cf. DIW (note 24), pp. 56-57.

${ }^{30}$ Cf. SCHNEILIN, SCHUMACHER (note 10), p. 76.

${ }^{31}$ Cf. DIW (note 27), pp. 56-57.

${ }^{32} \mathrm{Cf}$. Michel HAU, Histoire économique de l'Allemagne, XIX $-X X^{e}$ siècles, Paris, 1994, p. 210.

${ }^{33}$ Cf. TIMMERMANN (note 18), p. 17.

${ }^{34}$ Idem. 
au-delà de la planification centralisée, affirme la «propriété socialiste» des moyens de production ${ }^{35}$, autrement dit, une nationalisation quasi-totale.

Ce système économique est basé sur l'idéologie marxiste-léniniste. L'État socialiste contrôle tous les domaines de la vie politique, économique et sociale, pour des intérêts définis en théorie par la classe ouvrière, en réalité par les dirigeants du Parti communiste. La loi économique est pensée comme étant au service de la majorité et donc très supérieure en cela au capitalisme, et elle est présentée comme un processus scientifique et socialiste ${ }^{36}$.

Le principe de base en est un système de planification économique centralisé $^{37}$, sur le modèle soviétique. La direction de l'économie nationale est confiée, selon la constitution, au Conseil des ministres, qui charge de cette planification la Commission d'État au Plan, sous son contrôle. Des plans à long terme sont élaborés, sur cinq ou sept ans, et ils sont corrigés à la marge annuellement. Le plan annuel fixe le rythme de croissance de l'industrie, son volume et sa structure, tout comme celui de la main-d'œuvre et du commerce extérieur. Les ministères répartissent ensuite les programmes de production entre les différentes entreprises nationales et les combinats ${ }^{38}$. Les grandes entreprises, en coordination avec leurs sous-traitants et les autorités locales, élaborent alors un projet de plan annuel qui doit ensuite être approuvé par le Conseil des ministres. Les combinats sont chargés de coordonner ces plans pour un projet de branche, les ministères en font ensuite une synthèse par secteur et la Commission au Plan établit la synthèse globale. Une fois approuvé par le Conseil des ministres, le plan est retransmis jusqu'à l'échelon des entreprises sous la forme d'objectifs à réaliser obligatoirement ${ }^{39}$.

Les deux premiers plans quinquennaux (1951-1955 et 1956-1960) ont visé le développement des industries lourdes: industrie sidérurgique, industrie chimique, et production d'énergie hydraulique. Une première réorientation eut lieu en 1959, un nouveau plan de sept ans se superposant au plan quinquennal en cours. Il visait essentiellement à rattraper le retard accumulé par rapport à la RFA dans le domaine des biens de consommation, qui était estimé à 25-30\% en 1958. Mais il fut un échec et on y renonça dès $1961^{40}$.

Pendant près de 20 ans, le système de planification et d'organisation de l'économie est demeuré à peu près inchangé. Il se caractérisait essentiellement, en plus de la planification centrale de la production, par des

\footnotetext{
${ }^{35}$ Cf. SCHNEILIN, SCHUMACHER (note 10), p. 41.

${ }^{36}$ Idem.

${ }^{37}$ Voir le chapitre de Gabriele METZLER dans cet ouvrage.

${ }^{38}$ Les Vereinigung Volkseigener Betriebe (VVB) sont environ une vingtaine, à la tête, de fait, des branches industrielles. Ce principe est développé par la suite, et l'on en compte environ 120 à la fin des années 1980 ; cf. HAU (note 32), p. 210.

${ }^{39}$ Cf. Kurt SontheIMeR, Wilhelm BleEK, La République démocratique allemande, Paris, 1975, p. 183.

${ }^{40}$ Cf. SCHNEILIN, SCHUMACHER (note 10), pp. 76-77.
} 
prix durablement fixés, le devoir des entreprises de respecter la production planifiée et le monopole de l'État sur le commerce extérieur ${ }^{41}$.

L'année 1961 marque un tournant majeur pour la RDA, mais aussi pour son économie. En effet, avec la construction du mur, la RDA repart sur de nouvelles bases économiques, avec la fin de la saignée constante de la population active ${ }^{42}$. Cette importante perte a eu des conséquences majeures sur le développement économique de l'après-guerre, d'autant plus qu'une forte majorité de ces réfugiés étaient jeunes et le plus souvent professionnellement bien formés (techniciens, anciens entrepreneurs ou professions libérales) ${ }^{43}$. À partir de ce changement majeur de conditions, l'économie est-allemande a-telle pu retrouver une plus grande égalité avec celle de son homologue ouestallemande?

Depuis le milieu des années 1950, Walter Ulbricht et son conseiller économique, le Dr. Wolfgang Berger, étaient persuadés que la planification sur le modèle soviétique n'était pas la solution qui permettrait de dépasser l'économie ouest-allemande ${ }^{44}$. Certains estimaient qu'il y avait trop d'État et de bureaucratie. Des réformes économiques furent discutées et aussi en partie essayées, mais pour des raisons politiques, Ulbricht mit fin à ces discussions. Pour la majorité, dans le Politbüro les choses étaient simples : avec le mur, désormais, rien ne perturberait de l'extérieur et l'on pouvait désormais compter sur le fait que l'on travaillerait plus et que l'on consommerait moins. Ainsi avec plus de discipline, le Plan serait accompli. C'était la «petite solution» au problème économique de la RDA. Mais Ulbricht et Berger étaient partisans d'une « grande solution ${ }^{45}$.

Pour la préparation du $\mathrm{VI}^{\mathrm{e}}$ Congrès du SED (janvier 1963), le concept de réforme fut «retravaillé » par un petit groupe de l'équipe d'Ulbricht. «Les grandes lignes pour le nouveau système économique de la conduite et de la planification de l'économie sociale » $\left(\mathrm{NÖS}^{46}\right)$ furent présentées en juin 1963. L'économie ne devait pas être conduite dans un style autoritaire, mais avec l'art (science économique) et la manière.

Les insuffisances du système de production, telle que l'inadéquation du plan aux besoins nationaux ou la rigidité évidente du système du plan, entraînaient des conséquences économiques graves chaque jour plus

${ }^{41}$ Cf. DIW (note 27), pp. 56-57.

${ }^{42}$ La perte de population fut de 144000 à 330000 personnes par an, entre 1945 et 1961 . Alors qu'à la fin de la guerre, la population de l'Est était de 18 millions et représentait un quart de celle de l'Ouest, en 1976, elle n'en représente plus qu'un cinquième ; cf. GREGORY, LEPTIN (note 1), pp. 520, 525.

${ }^{43}$ GREGORY, LEPTIN (note 1), p. 522.

44 Jörg ROESLER, «Wirtschaftspolitische Grundsatzentscheidungen in beiden deutschen Staaten von 1946-1970», in : Utopie kreativ, 103/104 (mai-juin 1999), p. 105.

${ }^{45}$ Idem.

${ }^{46}$ Neues Ökonomisches System (NÖS). 
évidentes : lourdeur du système de production à l'intérieur des combinats qui générait une faible productivité, vieillissement des machines par suite d'une trop grande immobilisation des capitaux, pénuries fréquentes en raison des approvisionnements au sein CAEM et course à la quantité au détriment de la qualité $^{47}$.

L'idée novatrice fut alors la recherche d'une plus grande efficacité, en allégeant la bureaucratie rigide de la planification. Il fallait supprimer l'opposition entre les intérêts de croissance et de rentabilité, d'une part, et ceux des travailleurs et des entreprises, d'autre part. D'où une plus grande autonomie des entreprises dont la rentabilité fut soumise à la logique des coûts et des profits et qui purent disposer librement d'une partie de leurs bénéfices et créer des fonds propres (investissements, primes). Une réforme du système bancaire et une autre de la politique des prix fixés par l'État accompagna l'ensemble ${ }^{48}$.

Le NÖS donna par la suite ce que l'on attendait de lui : une stabilité économique, un niveau relativement élevé de taux de croissance de la productivité et de la production. Mais il ne permit cependant pas de dépasser ni même d'atteindre l'économie ouest-allemande ${ }^{49}$. Il se mua en "Système économique du socialisme » (ÖSS ${ }^{50}$ ) en $1967^{51}$ et fut prorogé jusqu'en 1971, avec une restriction des libertés économiques et plus de centralisation ${ }^{52}$. Erich Honecker remplaça Walter Ulbricht, démis de ses fonctions, en 1971, sur ordre de Moscou. Si Honecker reprit une partie de la réforme économique précédente, dans son ensemble, sa nouvelle politique imposa le retour au centralisme $^{53}$. Un des principaux changements consista dans le renforcement de l'intégration du CAEM (Komplexprogramm, 1971). Mais cette nouvelle réforme de 1971 est déjà morte en $1973^{54}$.

\section{Des objectifs assez parallèles}

$\mathrm{Au}$ moment où la rupture est consommée, avec la construction symbolique et pragmatique du mur de Berlin, les deux systèmes économiques sont déjà rodés depuis une longue décennie. S'ils radicalement différents dans leurs principes, leurs objectifs cependant révèlent des convergences, même si le poids

\footnotetext{
${ }^{47}$ SCHNEILIN, SCHUMACHER (note 10), p. 45.

${ }^{48}$ SCHNEILIN, SCHUMACHER (note 10), pp. 77-78.

${ }^{49}$ ROESLER (note 44), p. 106.

${ }^{50}$ Ökonomisches System des Sozialismus (ÖSS)

${ }^{51}$ C'est peut-être l'échec constaté en 1967 qui scelle définitivement le sort de l'économie est-allemande et qui débute son décrochage.

${ }^{52}$ TIMMERMANN (note 18), pp. 19-20.

${ }^{53}$ SCHNEILIN, SCHUMACHER (note 10), p. 79.

${ }^{54}$ Cf. Michael KEREN, «The New Economic System in the GDR: an Obituary », in : Soviet Studies, 24 (april 1973) 4, pp. 554-587.
} 
respectifs de certains éléments (croissance, efficacité économique, répartition équitable des revenus, augmentation du niveau de vie, qualité de l'environnement, stabilité économique, etc.) était différent en raison des choix politiques. Le principal objectif en RDA était le développement de l'industrie, et en RFA, plutôt la croissance des exportations et la stabilité des prix ${ }^{55}$.

Le livre de Werner $\mathrm{Obst}^{56}$, ancien haut dirigeant est-allemand ${ }^{57}$, montre bien l'obsession de la comparaison avec la RFA, pour le service des statistiques est-allemand. Mais côté ouest-allemand sont aussi publiées régulièrement des statistiques ${ }^{58}$ et plus récemment des livres d'analyse comparative $^{59}$. Il s'agit ainsi d'assurer le leadership du bloc dans le cadre de la guerre froide, et la propagande n'est pas la dernière des armes en la matière. Les très nombreuses statistiques produites par le gouvernement est-allemand étaient utilisées tant comme mécanisme de contrôle des objectifs du plan que pour montrer, à l'extérieur, les réalisations du régime ${ }^{60}$. De ce fait, les responsables étaient incités à surestimer la production, voire à inventer - selon certains, une véritable « économie fantôme » ${ }^{61}$. Ainsi, durant la Guerre froide, les pays à l'est de rideau de fer étaient tous soupçonnés de tenir des comptes doubles, l'un à destination du public, comme c'était le cas du livre annuel de statistiques publié en RDA, l'autre pour les dirigeants ${ }^{62}$. Cependant, on dispose des chiffres produits par les services de la RFA qui permettent une véritable comparaison. Du côté de l'Allemagne de l'Ouest, même si on nie la concurrence avec l'Est, les résultats économiques sont également mis en avant pour vanter la réussite du système.

En RDA, on considère que l'économie planifiée est l'opposée de l'économie capitaliste de marché, et pour cette raison elle doit être une réussite $^{63}$. Dès le premier plan $(1949 / 50)$ le système se pose en concurrence avec l'Ouest ( On verra quel système est le plus fort des deux ») ${ }^{64}$. Côté ouest, pour Erhard, le «collectivisme» est une abomination, mais en aucun cas un concurrent ${ }^{65}$.

${ }^{55}$ GREGORY, LEPTIN (note 1), p. 524.

${ }^{56}$ Werner OBST, DDR-Wirtschaft. Modell und Wirklichkeit, Hambourg, 1985.

${ }^{57}$ Responsable pour les questions économiques, qui a fui la RDA en 1969.

${ }^{58}$ Par ex. : Bundesministerium für Innerdeutsche Beziehungen (BIB), Zahlenspiegel. Ein Vergleich BRD-DDR, Bonn, 1970.

${ }^{59}$ Par ex. : Udo Wengst, Hermann WentKer (éd.), Das doppelte Deutschland. 40 Jahre Systemkonkurrenz, Berlin, 2008.

${ }^{60}$ Jaap SLEIFER, Planning ahead and falling behind. The East German economy in comparison with West Germany 1936-2002, Berlin, 2006, p. 29.

${ }^{61}$ Von der Lippe, cité par SLEIFER (note 60), p. 29.

${ }^{62}$ SLEIFER (note 60), p. 29.

${ }^{63}$ ROESLER (note 44), p. 102.

${ }^{64}$ ROESLER (note 44), p. 103.

${ }^{65}$ Idem. 
En 1950, l'industrie atteint à l'Est $75 \%$ de son niveau de 1936, alors qu'en RFA, on a déjà atteint $110 \%$ de ce niveau ${ }^{66}$. La croissance alors exceptionnelle en RFA constitue un aiguillon pour la RDA, mais les perspectives sont différentes. Alors qu'en RDA, les producteurs visent à remplir les objectifs de production fixés par le gouvernement », en RFA, les producteurs veulent atteindre le consommateur, ce qui génère des différences importantes dans la structure des produits et dans leur qualite ${ }^{67}$.

Par la suite, les écarts subsistent, mais certains domaines sont néanmoins assez comparables. En 1968, la valeur des biens industriels en RDA est de 140 milliards de Mark, soit $36 \%$ de celle de la RFA (389 milliards de Mark $)^{68}$. En ce qui concerne la part de la main d'œuvre dans l'industrie, il n'y a aucune différence entre les deux États [1971]. La production par poste de travail $^{69}$ en 1968 atteint presque 48000 Mark tant en RDA qu'en RFA (et en considérant la différence de niveau des prix du capital dans les deux pays, cette valeur doit être minorée de $15 \%$ pour l'Ouest $)^{70}$.

Si l'on compare la structure du capital industriel, on constate encore une grande convergence. Dans les deux pays, depuis 1960, plus de la moitié de la valeur brute du capital est placé dans l'industrie des matières premières. De même, dans l'industrie légère et dans l'industrie agro-alimentaire, la part y est presque identique (RDA : respectivement $15 \%$ et $8 \%$, RFA : $13 \%$ et $9 \%$ ). Dans l'industrie de transformation des métaux, on note cependant quelques différences : un peu moins de $20 \%$ en RDA, presque $25 \%$ en RFA (depuis le milieu des années 1960). Dans la répartition par branche industrielle, les écarts sont plus importants. En RDA, des secteurs tels que la construction, l'industrie chimique, la mécanique de précision et l'optique, tout comme certaines branches textiles et l'industrie du verre et de la céramique représentent une part des capitaux plus importante qu'en RFA. A l'inverse, ce sont les secteurs de l'énergie, de la métallurgie, de la construction mécanique et automobile et de l'électrotechnique qui représentent un poids supérieur à celui qu'il est en $\mathrm{RDA}^{71}$.

Mais d'une manière générale, l'écart entre les deux structures industrielles dans leur ensemble n'est pas très grand. Cependant, alors que jusqu'en 1960, on a pu constater un rapprochement entre les structures du capital, par la suite, les différences se sont à nouveau un peu accentuées ${ }^{72}$.

\footnotetext{
${ }^{66} \mathrm{Ibid}$.

${ }^{67}$ SLEIFER (note 60), p. 27.

${ }^{68}$ Valeur des prix de 1962 (à mettre en relation avec 17 millions d'habitants à l'Est contre $59,5 \%$ à l'Ouest, soit $28,6 \%$.

${ }^{69}$ Productivité du capital.

${ }^{70}$ DIW (note 27), pp. 46-47.

${ }^{71}$ Idem.

${ }^{72}$ Ibid.
} 
Les efforts des deux pays pour développer l'emploi industriel sont perceptibles et sont de même proportion et de même niveau avec une croissance de $42,7 \%$ à $48,2 \%$ à l'Ouest entre 1950 et 1969 , et de $43,9 \%$ à $49,0 \%$ à l'Est.

Tableau 1: Comparaison des emplois par secteur ${ }^{73}$

\begin{tabular}{|c|c|c|c|c|c|}
\hline \multirow[b]{2}{*}{ Secteurs économiques } & \multirow[b]{2}{*}{ Unité } & \multicolumn{2}{|c|}{ RFA } & \multicolumn{2}{|c|}{ RDA } \\
\hline & & 1950 & 1969 & 1950 & 1969 \\
\hline \multirow[t]{2}{*}{ Production totale } & 1000 pers. & 20376 & 26822 & 7196 & 7750 \\
\hline & $\%$ & 100 & 100 & 100 & 100 \\
\hline \multirow{2}{*}{$\begin{array}{l}\text { Secteur agricole, forestier et } \\
\text { pêche }\end{array}$} & 1000 & 5020 & 2533 & 2005 & 1026 \\
\hline & $\%$ & 24,6 & 9,5 & 27,9 & 13,2 \\
\hline Production manufacturière & 1000 pers. & 8689 & 12936 & 3163 & 3793 \\
\hline dont : & $\%$ & 42,7 & 48,2 & 43,9 & 49,0 \\
\hline \multirow[t]{2}{*}{$-\quad$ Industrie } & 1000 pers. & 6725 & 8308 & 2098 & 2823 \\
\hline & $\%$ & 33,0 & 31,0 & 29,2 & 36,4 \\
\hline \multirow[t]{2}{*}{ - construction } & 1000 pers. & 1116 & 1505 & 465 & 559 \\
\hline & $\%$ & 5,5 & 5,6 & 6,5 & 7,2 \\
\hline \multirow[t]{2}{*}{ Commerce et transport } & 1000 pers. & 2918 & 4729 & 1129 & 1419 \\
\hline & $\%$ & 14,3 & 17,6 & 15,7 & 18,3 \\
\hline \multirow[t]{2}{*}{ Autres secteurs et services } & 1000 pers. & 3749 & 6624 & 899 & 1512 \\
\hline & $\%$ & 18,4 & 24,7 & 12,5 & 19,5 \\
\hline
\end{tabular}

En RDA, la croissance de la part de l'industrie dans la formation du revenu national, forte dans le premier après-guerre (de $47 \%$ en 1950 à $56,4 \%$ en $1960)$ se poursuit jusqu'en 1971 (61,4\%), puis se stabilise. Cette croissance s'est faite au détriment de l'agriculture avant tout, mais aussi des transports ${ }^{74}$. Le développement de l'emploi total en RDA, malgré la saignée de l'émigration, s'explique par le renforcement de l'emploi féminin. En effet, malgré la même très forte orientation de la production sur l'industrie, les deux pays présentent des différences très importantes en termes d'emplois des femmes. Celles-ci sont bien plus nombreuses à travailler en RDA, et

\footnotetext{
${ }^{73}$ BIB (note 58), p. 28.

${ }^{74}$ Notes et études documentaires, 1973, p. 19, cité par SONTHEIMER, BLEeK (note 39), p. 174.
} 
l'accroissement régulier de ce travail féminin, sur la période, ne comble pas les écarts, bien au contraire. Ainsi, alors qu'en 1960, 51,3\% des femmes ouest-allemandes travaillent contre $61,9 \%$ des femmes est-allemandes, les écarts se sont creusés puisque qu'en 1969 elles ne sont que 52,9\% à travailler contre $74 \%$ à l'Est ${ }^{75}$.

Réussites et limites d'une croissance économiques

Les statistiques comparatives sont nombreuses pour la période 1961-1974. Même si celles provenant de la RDA ont été normalisées pour être comparables, il faut cependant faire remarquer que leur fiabilité n'est pas assurée $^{76}$. Cependant, ces données permettent des comparaisons pour les grandes lignes des évolutions économiques. Et elles montrent que tant la RFA que la RDA sont les pays qui ont connu les plus grosses réussites, pour chacun des systèmes auxquels elles adhèrent, ce qui est un des intérêts de leur comparaison ${ }^{77}$.

D'une manière générale, on peut considérer que les économies est et ouestallemande ont progressé à un taux de croissance proche entre 1960 et 1973, autour de $4,5 \%$ en moyenne par an, et on ne constate pas de différences majeures pour la croissance par secteur ${ }^{78}$. Malgré la priorité mise sur l'industrie en RDA, le secteur industriel a cru légèrement plus vite en RFA ; par contre le secteur de la construction s'y est développé un peu plus vite, et à l'opposé, le secteur des services un peu moins vite. Mais la similarité des deux croissances a été remarquable au cours de cette période. La principale différence est que le taux de croissance a été bien plus régulier en RDA (3,3 à $6,7 \%$ ) alors qu'en RFA les variations de cette croissance étaient plus marquées $(-2,4 \% \text { à }+11,5 \%)^{79}$, ce pays ayant subi à plusieurs reprises des fluctuations cycliques importantes. Cette plus grande stabilité économique estallemande (à la fois de la croissance, mais aussi l'absence d'inflation excessive et de chômage) a eu un coût considérable pour l'État est-allemand. Mais elle a eu des conséquences très positives pour la société est-allemande, puisque que les prix à la production et à la consommation ont augmenté moins

\footnotetext{
${ }^{75}$ D'après les données du BIB (note 58), p. 28.

${ }^{76}$ GREGORY, LEPTIN (note 1), p. 525, note 23 et annexe 1: "problems of statistical comparisons of east and West Germany », pp. 539-540.

${ }^{77}$ GREGORY, LEPTIN (note 1), p. 525.

${ }^{78}$ GREGORY, LEPTIN (note 1), p. 526. De son côté, Werner Obst observe une croissance moyenne de 4,6 \% pour chacun des deux États entre 1961 et 1972, avec un niveau très stable (à l'exception de l'année 1962 avec 1,2 \%) pour la RDA, tandis que les années 1971 et 1972 sont déjà très difficiles pour la RFA avec seulement 2,8 \% de croissance en moyenne par an ; OBST (note 56), tableau 1, p. 17.

${ }^{79}$ GREGORY, LEPTIN (note 1), p. 526-527.
} 
vite qu'à l'Ouest pour cette période. L'indice officiel de la RDA indique même une diminution de $10 \%$ entre 1955 et 1973.

\section{Les expériences d'une première crise en RFA}

En RFA, la très bonne conjoncture de l'après-guerre dure plus longtemps qu'en RDA. La production industrielle croît de $150 \%$ entre 1950 et 1960 et depuis la fin des années 1950, on a atteint le plein emploi ; dès 1960, il y a même pénurie de main d'œuvre ${ }^{80}$. On fait donc appel à l'immigration et en 1964, on compte plus d'un million de travailleurs étrangers qui viennent compléter le réservoir national de main d'œuvre (à la fin des années 1960, atteint plus de 2 millions) ${ }^{81}$. En 1961, sur 686000 personnes, ce sont encore les populations immigrées venues d'Italie, puis d'Espagne et de Grèce qui dominent. En 1970, les Turcs sont devenus la troisième communauté derrière les Italiens et les Yougoslaves, puis la première en 1973 (893000 personnes) ${ }^{82}$. Ainsi, la croissance très forte de la population ouest-allemande ( $+23,3 \%$ en 20 ans depuis la création de la RFA), au-delà de l'accroissement naturel, s'explique par le solde migratoire fortement positif, avec l'apport des réfugiés de RDA auxquels s'ajoutent les migrants du travail du Sud de l'Europe et de Turquie. Ceci a constitué un atout essentiel pendant la période de croissance, jusqu'en 1973.

Des records de croissance avaient été atteints en 1964 en RFA, mais on craignait, dès 1965, une crise, et celle-ci survient l'année suivante (1966) avec un fort recul du taux de croissance. L'augmentation des prix entraine une baisse du pouvoir d'achat, et à la fin de l'année 1966, le chômage dépasse 500000 personnes. Ce retournement de la conjoncture s'explique en partie par la fin du «boom» économique de l'après-guerre et la saturation du marché national (demande satisfaite) ${ }^{83}$. Les taux de croissance de la RFA étaient alors au dessus du niveau mondial et inévitablement ils devaient baisser. Mais cette évolution inéluctable n'avait pas été anticipée ni par les dépenses publiques, ni par les investissements. Le problème était alors que cette croissance ralentie ne se transforme pas en une véritable dépression. Mais, pour l'éviter, beaucoup, dont une fraction de la CDU, réclamaient une « intervention intelligente » de l'État, ce à quoi le gouvernement en place se refusait. Les mesures habituelles de la Bundesbank ne furent pas efficaces cette fois-ci et le chômage augmenta, tout comme le niveau d'inflation, et la croissance faiblit beaucoup. La politique économique du chancelier Erhard (1963-1966), est ainsi largement

${ }^{80}$ Cf. ROESLER (note 44), p. 107.

${ }^{81}$ Cf. Harald WINKEL, Die Wirtschaft im geteilten Deutschland 1945-1970, Wiesbaden, 1974, p. 75.

${ }^{82}$ Cf. SCHNEILIN, SCHUMACHER (note 10), p. 49.

${ }^{83}$ Cf. WINKEL (note 81), p. 76. 
décriée, car il nie l'évidence de la crise conjoncture, jusque tard dans l'année 1966.

Le SPD décide alors de favoriser un départ d'Erhard et commence à négocier avec le FDP. À la CDU, cela mit en route le signal d'alarme et un jeu d'intrigue interne visa à forcer le départ d'Erhard qui se retira le 10 novembre 1966. La CDU débuta alors des négociations avec le SPD, ce qui déboucha sur la formation de la Grande Coalition (26 novembre 1966) qui proposa enfin des solutions à la crise ${ }^{84}$. Une série de mesures importantes fut annoncée par la loi de stabilite ${ }^{85}$ du 10 mai 1967. Ce fut une réussite et les années suivantes, la croissance redevint satisfaisante (le taux de croissance du PNB passe de $0,1 \%$ en 1967 à $6,1 \%$ en 1968 , puis à $7,5 \%$ en 1969) et le chômage baissa $(1,5 \% \text { en } 1968,0,9 \% \text { en } 1969,0,7 \% \text { en } 1970)^{86}$. L'inflation elle-même semble maîtrisée (de $3,5 \%$ en 1966 à $1,7 \%$ en 1967 et 1968). Ces succès économiques durèrent jusqu'en $1970^{87}$.

La récession de 1966/67 a cependant été un véritable choc pour la RFA, avec des conséquences durables. On réalisa que cette récession n'était pas une exception, mais que bien au contraire elle constituait la règle des États industrialisés occidentaux ${ }^{88}$. Une légende avait ainsi été détruite, celle de l'économie de marché qui évite les crises et permet une constante élévation du niveau de vie, et la stabilité politique de longue durée de la RFA fut remise en question ${ }^{89}$. On prit conscience que la « société de consommation sans classe » n'existait pas et qu'au contraire, on renouait avec les problèmes classiques de surproduction et de chômage. Il s'agit donc d'un tournant fondamental pour la société allemande.

Malgré la réussite économique générale, la croissance n'allait pas tout-à-fait sans problème. Ceci est ressenti une première fois en 1958, quand l'économie charbonnière subit une crise structurelle en raison de la concurrence du pétrole et des produits importés, moins chers. Alors que dix ans plus tôt, la chose était inimaginable, sept millions de tonnes de charbon restent invendues. Une aide de l'État (1962) fut nécessaire pour prendre des mesures de rationalisation ${ }^{90}$.

Or, après la crise du charbon, le secteur de l'industrie lourde est mis à son tour en difficulté dès le milieu des années 1960. Le 9 juin 1967, à l'initiative de l'industrie, un «Rheinstahl-Plan » est proposé au ministre de l'économie ${ }^{91}$. Par la suite, des négociations furent organisées, avec la participation de la « IG

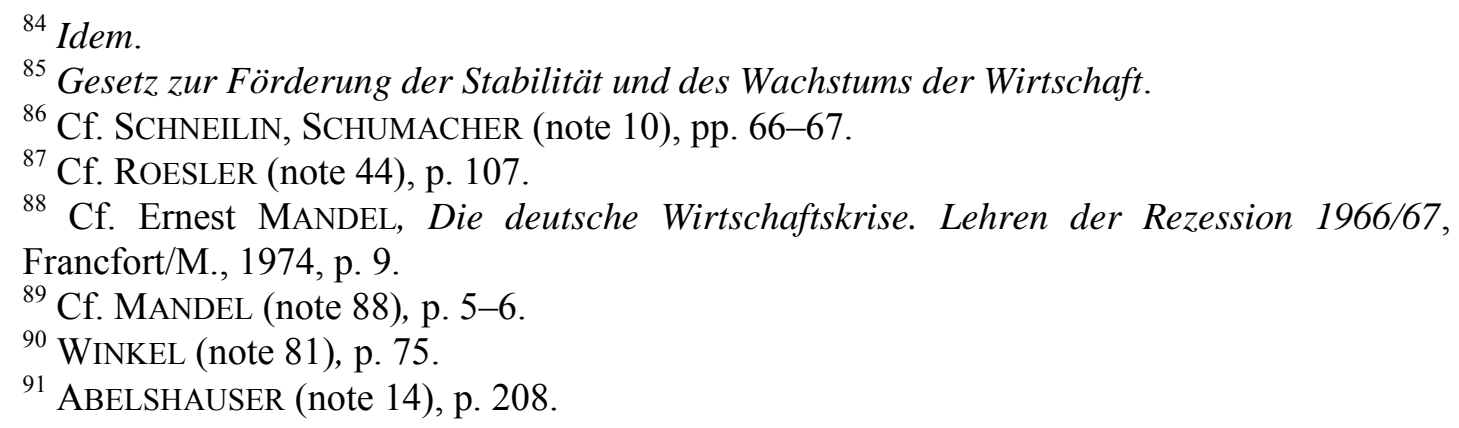


Bergbau und Energie» (IGBE). Une société unique fut créée («Ruhrkohle AG ») avec une structure de « cogestion ${ }^{92}$. Cette création mit fin à la crise en 1969. Le plan prévoyait dans les cinq ans la fermeture de 19 puits (passage de 52 à 33), la réduction du personnel de 183000 à 150 000, et également l'arrêt de huit cokeries, sans conflit social et sans chômage. Le but de cette réorganisation et de la concentration en une seule société était la consolidation des entreprises minières de la Ruhr qui, depuis dix ans, n'avaient pas de politique entrepreneuriale ni énergétique commune. Environ 15 milliards de marks d'aides publiques, dont au moins 10 milliards rien que pour l'industrie minière, furent dépensés, sans effet car la situation ne fut pas sauvée de manière définitive et, de 1970 à 1978, il y eut à nouveau près de 10 milliards de marks de subvention ${ }^{93}$.

Après la fin de la crise, en 1967, et jusqu'au choc pétrolier de 1973, la croissance économique reprend ( $5 \%$ de croissance du PNB en moyenne), avec cependant une forte hausse des dépenses publiques (de 34,2\% du PNB en 1961 à 49,5\% en 1975) ${ }^{94}$. En effet, sous l'influence de Karl Schiller (SPD), ministre de l'économie de 1966 à 1972, le gouvernement ouest-allemand modifie la voie traditionnelle de l'ordolibéralisme allemand en y introduisant de fortes inflexions keynésiennes. Ceci marque, pour la RFA, le début d'une nouvelle ère de politique économique : le principe de l'ordolibéralisme est complété par l'apport d'éléments de planification de branches sectorielles ${ }^{95}$.

L'ouverture sur les marchés extérieurs se poursuit (sur la période, 10,6\% de croissance des importations et $8,7 \%$ des exportations) et la balance commerciale est de plus en plus excédentaire (16,9 milliards de DM en 1967 ; 37,3 en 1973). Mais l'industrie voit reculer sa part dans le PNB (de 54,5\% en 1961 à $47,4 \%$ en 1975) ${ }^{96}$, même si la production industrielle continue de croître à un rythme moyen annuel de $6,4 \%$.

Les salaires et les prix recommencent aussi à augmenter, mais l'emploi stagne ou presque $(0,6 \%$ de croissance annuelle moyenne entre 1967 et 1973). La part du chômage a ainsi crû sur la longue durée $(0,7 \%$ en $1961 ; 1,8$ en $1967 ; 4,2$ en 1975) ${ }^{97}$. La lutte contre l'inflation (les prix à la consommation augmentent de 4,1\% en moyenne annuelle entre 1967 et 1973) est au centre de toutes les politiques économiques du début des années 1970. Avec la deuxième réévaluation du Mark (24 octobre 1969) et les multiples programmes de stabilisation (juillet 1970 et mai 1971), on veut prolonger les

\footnotetext{
${ }^{92}$ Mitbestimmung; in ABELSHAUSER (note 14), p. 209.

${ }^{93}$ ABELSHAUSER (note 14), pp. 210-211.

${ }^{94}$ SCHNEILIN, SCHUMACHER (note 10), p. 64.

${ }^{95}$ WINKEL (note 81$)$, p. 76.

${ }^{96}$ SCHNEILIN, SCHUMACHER (note 10), pp. 64-66.

${ }^{97}$ SCHNEILIN, SCHUMACHER (note 10), p. 64.
} 
éléments de stabilité (prix stables, lutte contre l'inflation, plein emploi, équilibre des comptes et une croissance raisonnable $)^{98}$.

Le contrôle de la conjoncture s'avère cependant complet à la fin des années 1960, avec une économie de plein emploi, fortement liée à l'économie mondiale et hautement industrialisée, plus que dans les années de reconstruction ${ }^{99}$. Le système social de marché est redevenu un modèle de réussite. On reprend alors les discussions sur les questions de salaires et sur la cogestion (qui avaient cessé lors de la crise de 1966/67). Déjà dans les années 1960, le but de la politique sociale était la "propriété pour tous » et des évolutions de salaires. Certains résultats avaient été obtenu: à côté de nombreux allègements de taxes et de mesures en faveur de l'épargne, la mise en place d'actions populaires, entre 1959 et 1965, de participations (pour le personnel) depuis 1959, une loi sur la capitalisation (à destination des travailleurs, du 12 juillet 1961, et celle du 5 mai 1965). Ces questions revenaient donc au centre des préoccupations au début des années $1970^{100}$.

Les évolutions économiques ont eu des conséquences importantes sur la société allemande. Ceci est traité dans un autre chapitre, mais on peut simplement évoquer ici un processus frappant dans la société ouest-allemande des années 1950 et 1960 : celui de la « déprolétarisation » ${ }^{101}$ des travailleurs, autrement dit la fin du prolétariat. Elle est due à une meilleure formation, à des salaires plus élevés (le salaire réel a plus que doublé dans les années 1950) et payés mensuellement sur un compte, etc. Ceci est perceptible dans le changement des dénominations de ces travailleurs, qui deviennent plus communément des «Arbeitnehmer» ou des «Mitarbeiter » ${ }^{102}$ que des «Arbeiter » ou « Angestellte ${ }^{103}$.

\section{Croissance rapide, mais faible productivité en RDA}

De son côté, la RDA a connu une croissance économique rapide dans les années 1960, en particulier dans le secteur industriel qui était son objectif majeur ${ }^{104}$. Un des seuls aspects négatifs est celui de la productivité du travail. Dans ce domaine, et par rapport à la situation de l'avant-guerre, la RDA aurait eu un retard de $50 \%$ par rapport à la RFA. Cet écart a tendu à se combler légèrement pendant les années 1960, mais il est resté d'au moins un tiers et après 1967, il n'a plus réussi à se réduire ${ }^{105}$.

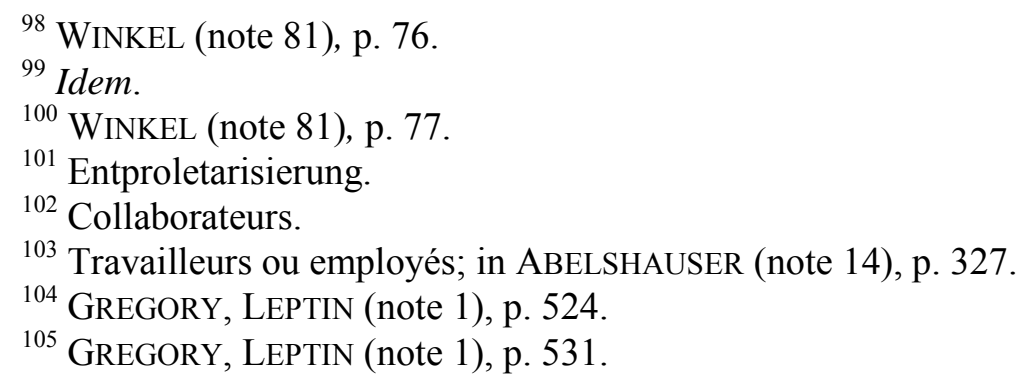


Après les succès des années 1957 et 1958, avec une croissance respective de $7 \%$ puis $12 \%$, les dirigeants avaient espéré rattraper le retard par rapport à la RFA dès 1961. Mais les mauvaises prévisions et planifications conduisirent à un repli de la croissance ( $6 \%$ en 1960, $4 \%$ en 1961) et les difficultés d'approvisionnement furent combattues par des mesures de collectivisation (1960). Il s'ensuivit une augmentation des flux vers l'Ouest. De plus, les investissements stagnèrent, l'ouverture de nouvelles capacités de production fut retardée et les pénuries de matières premières et de demi-produits ralentirent la production dans de nombreuses unités. On chercha alors à gagner sur la productivité du travail en augmentant les normes et en faisant stagner les salaires, afin de trouver une solution à cette crise de la croissance ${ }^{106}$.

Les dirigeants étaient bien conscients de ces difficultés et en 1961 les objectifs de production furent révisés, puis un nouveau Plan «prospectif » fut mis en place en 1962 (dans la perspective de 1970). L'échec du $7^{\mathrm{e}}$ Plan avait mis en évidence l'inadéquation des méthodes de planification (« idéologie de la tonne » qui conduisait à de mauvais investissements), de la fixation des prix (qui avait des conséquences graves pour le gaspillage des matières premières et bloquait l'innovation technologique) et de l'organisation économique (la «surcentralisation» des décisions empêchait tout intérêt, toute initiative et toute responsabilisation dans les usines) ${ }^{107}$. Lors de la phase des réformes (1962-1971), dans le cadre de la NÖS, on visa particulièrement à alléger les rigidités du système ${ }^{108}$.

Les difficultés réapparurent à la fin des années 1960, en particulier dans le domaine des transports et de l'approvisionnement en énergie. Mais ce fut avant tout une décision politique qui contribua au remplacement de Walter Ulbricht par Erich Honecker, plus proche des orientations soviétiques. Ceci eut pour conséquence l'abandon des réformes et le retour au centralisme et les relations au sein du CAEM et avec l'URSS furent renforcées ${ }^{109}$. Ainsi, la lourdeur d'une planification rigide, qui reprit de plus belle, ralentit la production est-allemande et les écarts augmentèrent (croissance industrielle et productivité).

\section{Les spécificités du commerce extérieur}

Le bouleversement territorial et politique subi par l'Allemagne après-guerre a eu des conséquences encore plus fortes dans le domaine du commerce extérieur. La rupture du territoire, doublée d'une rupture politique, transforme brutalement les flux traditionnels d'approvisionnement. Ainsi, l'est de

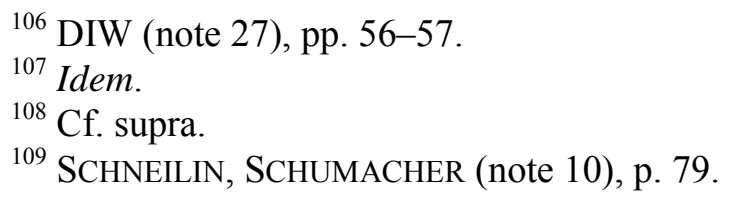


l'Allemagne n'est plus là pour assurer l'approvisionnement agricole de l'Ouest. Alors que sous le Reich, la part des importations était de $20 \%$, elle atteint désormais $50 \%$ à l'ouest. Le partage de l'Allemagne a des conséquences identiques sur les structures industrielles et même l'organisation de la politique commerciale en est touchée. Tous les anciens accords bilatéraux (est et sud-est) ont été coupés par le mur de fer ${ }^{110}$. Après les réorganisations nécessaires et le nouveau fonctionnement au sein des deux blocs (CAEM et CEE), pendant les années 1950, le commerce extérieur profite de la croissance générale.

Au cours des années 1960, la croissance du commerce extérieur a été importante, dans les deux pays (même si elle reste inférieure à la croissance globale). Ainsi, pour la RFA, entre 1960 et 1969, le total des importations est multiplié par 2,29 en valeur, celui des exportations par 2,37, tandis qu'en RDA, pour la même période, le total des importations est multiplié par 1,84 en valeur, celui des exportations par 1,92.

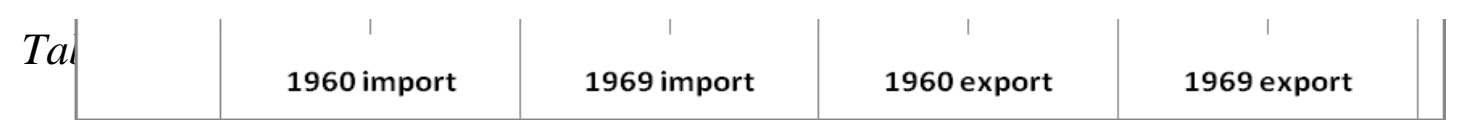

On assiste, pendant les années 1950 et 1960, à une certaine modification de la composition des échanges de la RFA. Les importations de produits alimentaires passent de 44,1\% (1950) à 26,3\% (1960) et 19,1\% (1970). Tandis que la part des importations de produits industrialisés finis passent de $12,6 \%$ (1950) à 32,2 (1960) puis à $50 \%$ (1970). Pendant ce temps, les exportations se modifient aussi : la part des matières premières chute de $14 \%$ (1950) à 1,9\% (1960), celle des produits finis passe de 64,9\% (1950) à $83,4 \%$ (1980). Donc la réintégration de la RFA dans le marché mondial profite beaucoup à sa production industrielle ${ }^{112}$, en particulier à quelques branches industrielles telles que la construction mécanique, les systèmes de télécommunication, l'électroménager et les véhicules ${ }^{113}$.

Dans le domaine des échanges extérieurs, la RDA ne soutient pas vraiment la comparaison. Elle se situe environ à $50-60 \%$ du volume des échanges par habitant de la RFA. Cette tendance autarcique n'a pu avoir que des conséquences négatives sur la croissance et les performances de la productivité, d'autant plus que cette économie était mal armée pour l'autosuffisance ${ }^{114}$.

\footnotetext{
${ }^{110}$ Abelshauser (note 14), p. 219.

${ }^{111}$ D'après les données du BIB (note 58), p. 34.

${ }^{112}$ ABELSHAUSER (note 14), p. 262.

${ }^{113}$ ABELSHAUSER (note 14), p. 233.

${ }^{114}$ GREGORY, LEPTIN (note 1), p. 523.
} 
Alors que dans le premier après-guerre, la RFA a plutôt été la bénéficiaire de technologies avancées, la RDA a, de son côté, plutôt joué le rôle d'exportatrice de technologie au sein du bloc communiste ${ }^{115}$, en raison de son avance sur les autres pays (à l'exception de l'URSS). Cette situation de domination dans son aire économique a également probablement contribué à une faible pression de la concurrence, ce qui évidemment ne pousse pas à l'innovation.

La structure de ce commerce extérieur est totalement différente entre les deux pays. Le commerce extérieur est-allemand est orienté vers les autres pays socialistes, en particulier l'URSS, via le Conseil d'Assistance Économique Mutuelle (CAEM) ${ }^{116}$, créé le 25 janvier 1949 à Moscou comme un pendant au Plan Marshall et à l'OECE. La RDA y est admise dès 1950. La part de ses échanges avec les États du bloc communiste est de 80,6\% pour les importations et 78,2\% pour les exportations, en 1969. Côté RFA, ces chiffres sont respectivement de $4,1 \%$ et de $4,5 \%$. Avec la nouvelle situation issue de la guerre, on a vu une chute presque totale des exportations de la RFA vers l'ensemble de l'Est européen (1 à $2 \%$ seulement dans années 50), situation renforcée par l'embargo (CoCom-Liste) ${ }^{117}$. Sous ces conditions, les échanges avec l'Est et le sud-est européens n'atteignent que $5 \%$, même si avec ce chiffre, la RFA est largement en tête des échanges de l'ouest avec ces régions ${ }^{118}$. Cependant, si l'on prend en considération l'ensemble des échanges allemands, la répartition n'a guère changé par rapport à celle de l'avant-guerre $(15 \% \text { des exportations avec Est et Sud-est })^{119}$

L'entrée en vigueur de la Communauté économique européenne (CEE), à partir de 1958, a été certainement un élément de renforcement de l'intégration de l'Allemagne à l'Ouest au sein de l'Europe occidentale. A cette date, l'Allemagne exportait $27 \%$ vers la zone CEE, en 1971, ce pourcentage atteignait $40 \%$, et dès le premier élargissement (1973) cette part était de plus de $50 \%{ }^{120}$.

La forte insertion de la RFA dans le marché mondial n'a cependant pas eu que des avantages. Dans les années 1960 et 1970, le bilan commercial, constamment excédentaire, conduit à un transfert de biens à l'étranger et à une accumulation de réserves monétaires pas toujours très productives. La

\footnotetext{
${ }^{115}$ Idem.

${ }^{116}$ Rat für gegenseitige Wirtschaftshilfe (RGW).

117 CoCom : Coordinating Committee for Multilateral Strategie Export Controls (la RFA n'en fait pas partie aux débuts, car elle n'est pas encore membre de l'OTAN, mais elle en suit les directives). Il s'agit d'une liste de produits sous embargo dans le cadre du commerce est-ouest (à partir de 1951), en particulier machines, ordinateurs et produits qui peuvent être utilisés à des fins militaires.

${ }^{118}$ ABELSHAUSER (note 14), p. 261.

${ }^{119}$ Idem.

${ }^{120}$ ABELSHAUSER (note 14), pp. 260-261.
} 
tendance inflationniste est en partie due au commerce extérieur (inflation importée, car le niveau de prix à l'étranger est supérieur). Et de plus, l'excédent de la balance commerciale ouest-allemande entraîne, dans les pays déficitaires des mesures de rétorsion ${ }^{121}$.

Dans le domaine du commerce extérieur, les deux systèmes sont évidemment organisés très différemment. En RDA, le commerce extérieur est une prérogative de l'État, en RFA, l'État a pris un rôle d'orientation et a mis en place un cadre normatif ${ }^{122}$. En RDA, le ministère du commerce extérieur dirige, organise, planifie et contrôle entièrement le commerce extérieur sur la base de décision du parti et du gouvernement. C'est un monopole d'État (art. 9.5 de la Constitution) qui s'opère à l'aide d'organisation et d'institutions spécifiques, parmi lesquelles des représentations commerciales dans les pays tiers, de chambres de commerce extérieur, un bureau du commerce extérieur, des entreprises spécifiques, enfin même un institut de recherche dépendant du ministère. L'appartenance de la RDA au Pacte de Varsovie et au CAEM ainsi que le système bilatéral des échanges dans les pays de l'Est font que $70 \%$ de ce commerce s'effectue avec les pays du bloc de l'Est ${ }^{123}$. Mais les résultats globalement très insuffisants font que le commerce extérieur a été considéré comme l'une des raisons pour lesquelles la RDA restait économiquement largement en recul de la RFA ${ }^{124}$ ? C'était son «talon d'Achille » ${ }^{125}$ dans la compétition économique avec l'Ouest.

Les échanges entre les deux Allemagne sont, dans les premiers temps de leur existence, presque interrompus. Par la suite, malgré la position officielle de refus prise par la RFA, ces échanges ont cependant commencé à croître. Le protocole du traité de la CEE relatif au commerce interallemand prévoit que les échanges entre la RFA et la RDA ne seront pas touchés par le tarif extérieur commun ${ }^{126}$. Ces échanges ont lieu par l'intermédiaire d'organismes privés auxquels le gouvernement ouest-allemand a donné un monopole dans ce domaine ${ }^{127}$. Ainsi, le gouvernement de la RFA peut officiellement esquiver la réalité de ces échanges, tout en gardant un droit de regard et la RDA peut

\footnotetext{
${ }^{121}$ ABELSHAUSER (note 14), p. 263.

${ }^{122}$ TIMMERMANN (note 18), pp. 23-24.

${ }^{123}$ Idem.

${ }^{124}$ ROESLER (note 44), p. 105.

${ }^{125}$ Christoph BuCHHEIM, «Die Achillesferse der DDR - der Außenhandel », in : André STEINER (éd.), Überholen ohne einzuholen. Die DDR-Wirtschaft als Fußnote der deutschen Geschichte?, Berlin, 2006, pp. 91-103.

${ }^{126}$ Marie-Thérèse BITSCH, Histoire de la construction européenne de 1945 à nos jours, Bruxelles, 1996, p. 122.

${ }^{127}$ Chantal METzGER, «La vision française de la RDA de 1949 à 1955 », in : Elisabeth DU RÉAU (éd.), Regards croisés et coopération en Europe au XX siècle, Paris, 1996, p. 156.
} 
ainsi être le "passager clandestin » de la $\mathrm{CEE}^{128}$ et bénéficier d'un statut préférentiel (taxes). A partir de l'Ostpolitik (1969) et plus encore, du traité fondamental (1972), qui prévoit une «amélioration des échanges », ceux-ci s'accélèrent et la RFA devient le second partenaire commercial de la RDA derrière l'URSS ${ }^{129}$.

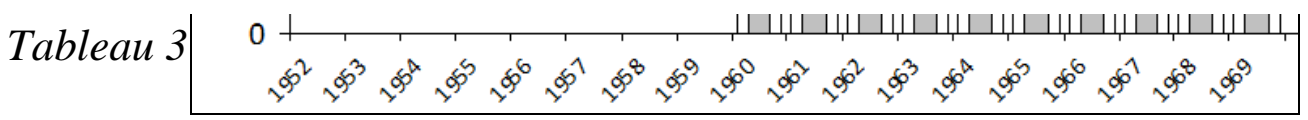

Cependant, si cette normalisation permet de renforcer les échanges, elle n'empêche pas la poursuite de la concurrence économique entre les deux États. Par ailleurs, du fait de la progression constante des échanges, on peut y voir une captation progressive de l'économie est-allemande par l'essor de la RFA $^{131}$.

En ce qui concerne la structure de ces échanges, on constate, au cours des années 1960, quelques évolutions. Ainsi, plus de la moitié des exportations de la RFA vers la RDA relève des matières premières et des biens de production, et cette part augmente encore (de 52 à $55 \%$ ), alors que, pour les mêmes catégories, les importations chutent de moitié (de 55 à $28 \%$ ). Au contraire, pour ce qui relève des biens d'équipement, les importations se renforcent (de 10 à $15 \%$ ) tandis que les exportations s'affaiblissent (de 26 à $22 \%$ ). Pour les biens de consommation, les exportations ouest-allemandes restent à peu près fixes (de 9 à $8 \%$ ), mais les importations augmentent nettement (de 19 à $33 \%$ ), tout comme pour les produits alimentaires (de 16 à $26 \%$ ). Ceci reflète les évolutions de la production dans les deux pays.

Tableau 4 : La structure du commerce entre les deux Allemagne ${ }^{132}$

${ }^{128}$ Le Monde, 24 janvier 1973, cité par Dorothee RöSEBERG, Images de la France en République démocratique allemande: une histoire oubliée, Paris, 2004, p. 19.

${ }^{129}$ Peter Christian LUDZ, Deutschland doppelte Zukunft. Bundesrepublik und DDR in der Welt von morgen, Munich, 1974, pp. 163-164.

${ }^{130}$ D'après les données du BIB (note 58), p. 36 et du DIW (note 27), p. 234. Les effets des deux crises en RFA sont très visibles.

${ }^{131}$ Pierre-Frédéric WEBER, Le triangle RFA-RDA-Pologne (1961-1975). Guerre froide et normalisation des rapports germano-polonais, Paris, 2007, p. 282.

${ }^{132}$ DIW (note 27), p. 233. 


\begin{tabular}{lcccc}
\hline & \multicolumn{2}{c}{$\begin{array}{c}\text { Exportations } \\
\text { de la RFA en \% }\end{array}$} & \multicolumn{2}{c}{$\begin{array}{c}\text { Importations } \\
\text { de la RFA en \% }\end{array}$} \\
\hline & 1960 & 1969 & 1960 & 1969 \\
Matières premières et biens de production & 52 & 55 & 55 & 28 \\
$\quad$ dont : chimie & 15 & 21 & 8 & 9 \\
Biens d'équipement & 26 & 22 & 10 & 15 \\
$\quad$ dont : construction de machines & 15 & 16 & 7 & 7 \\
Biens de consommation & 9 & 8 & 19 & 33 \\
Produits agricoles et alimentaires & 12 & 15 & 16 & 25 \\
\hline
\end{tabular}

Comparaison, compétition ou convergence

Au cours des années 1960, dans les deux pays, les mécanismes économiques jouent un rôle important. La coordination s'effectue soit par le marché et les prix, soit par la planification, la stimulation et le contrôle soit par la concurrence ou par des pressions économiques ${ }^{133}$. On y constate une très forte concentration des entreprises, de la production et du pouvoir économique, mais ils ne sont pas dans les mêmes mains. En termes de résultats, la productivité du travail, le produit social et le niveau de vie sont en RDA à peu près les deux tiers de celui de la RFA ${ }^{134}$.

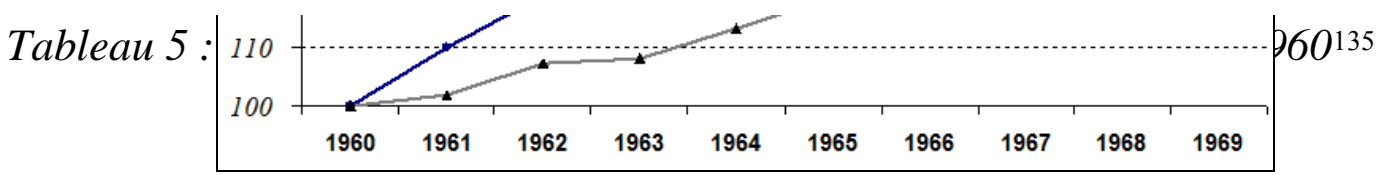

La comparaison des indices confirme le creusement de certains écarts entre les deux Allemagne au cours des années $1960^{136}$, comme c'est le cas pour le PNB (revenu global) ou bien pour la productivité industrielle qui, au cours des années 1960, progresse bien plus côté RFA que côté RDA (48,4\% contre $32,5 \%$ ). Mais les évolutions sont inégales, avec un recul est-allemand dans l'industrie lourde, mais une bonne tenue dans le secteur alimentaire et des gains comparatifs dans les industries légères et métallurgique, et au total un

\footnotetext{
${ }^{133}$ TIMMERMANN (note 18), p. 24.

${ }^{134}$ Selon TIMMERMANN (note 18), une des explications en est le problème de la motivation.

${ }^{135}$ Indices calculés d'après les données du BIB (note 58), p. 26.

${ }^{136} \mathrm{~A}$ la réserve près qu'il n'a pas été possible de déterminer si les données statistiques étaient en marks constants. Si ce n'est pas le cas, les variations seraient sans doute atténuées, car l'inflation en RFA (moyenne de 3,4 \% entre 1961 et 1973 - source OCDE), était sans doute supérieure à celle de la RDA (les prix étaient fixés par l'État et la plupart des matières premières étaient très largement à des prix en dessous des coûts de production jusqu'en 1964-1967 où les réformes ont relevé les prix, pour une meilleure adéquation avec les coûts; les prix industriels et des biens de consommation ont été réévalués à nouveau en 1973).
} 
écart qui se maintient dans la globalité (cf. tableau infra). Les années 1960 ne sont donc pas encore celles du décrochage de l'Est, bien au contraire, puisque le système est plutôt amélioré.

Tableau 6 : Productivité comparée RDA-RFA de 1960 à $1968^{137}$

\begin{tabular}{|c|c|c|c|c|c|c|}
\hline \multirow[b]{2}{*}{$\begin{array}{l}\text { Branches } \\
\text { industrielles }\end{array}$} & \multicolumn{2}{|c|}{$\begin{array}{c}\text { Productivité du travail } \\
\text { (1968) en DM } \\
\end{array}$} & \multicolumn{2}{|c|}{$\begin{array}{c}\text { Croissance (productivité) } \\
\text { de } 1960 \text { à } 1968 \text { en \% } \\
\end{array}$} & \multicolumn{2}{|c|}{$\begin{array}{c}\text { RDA en \% de } \\
\text { la RFA } \\
\end{array}$} \\
\hline & RDA & RFA & RDA & RFA & 1960 & 1968 \\
\hline $\begin{array}{l}\text { Industrie de base } \\
\text { (matières premières) }\end{array}$ & 51660 & 81020 & 51,8 & 77,9 & 74,8 & 63,8 \\
\hline $\begin{array}{l}\text { Industrie } \\
\text { métallurgique }\end{array}$ & 26170 & 40310 & 63,5 & 27,4 & 50,6 & 64,9 \\
\hline Industrie légère & 27880 & 40900 & 56,0 & 46,2 & 63,9 & 68,2 \\
\hline $\begin{array}{l}\text { Industries } \\
\text { alimentaires }\end{array}$ & 90120 & 113870 & 41,7 & 39,9 & 78,2 & 79,1 \\
\hline Industrie au total & 38460 & 56200 & 32,5 & 48,4 & 66,6 & 68,4 \\
\hline
\end{tabular}

Certaines caractéristiques du système économique socialiste apparaissent évidentes à la comparaison: moins d'investissement dans les transports et dans la construction de logement et une part un peu plus grande de l'industrie lourde dans la production totale. Celle-ci se renforce encore au cours des années 1960, en particulier dans l'industrie métallurgique dans laquelle la productivité du capital est proche en RDA (1366 DM en 1968) de celle de la RFA (1475 DM). Ainsi, dans ce secteur, la RDA comble progressivement l'écart, passant de 60,5 à 92,6\% par rapport à la productivité du capital en RFA. Plus encore, le secteur de l'industrie agro-alimentaire affiche une meilleure performance et le ratio de l'Est par rapport à l'Ouest passe de $108,7 \%$ en 1960 à $141 \%$ en 1968 . Le seul secteur dans lequel ce ratio ne s'améliore pas pour la RDA est celui des matières premières ${ }^{138}$.

D'une manière générale, si l'on s'en tient aux chiffres de la RDA, le taux d'investissement est-allemand aurait été inférieur à celui de la RFA, même si l'on note sur la fin de la période plutôt une évolution vers moins de différences dans ce domaine. Pourtant, en appliquant les critères de l'Ouest aux statistiques, la chose n'est plus certaine, et il semble même que sur la période examinée ici, les taux d'investissements aient même été plus élevés en $\mathrm{RDA}^{139}$. On peut sans doute en conclure que la différence était faible, et de

${ }^{137}$ DIW (note 27), p. 285.

${ }^{138}$ DIW (note 27), p. 287.

${ }^{139}$ GREGORY, LEPTIN (note 1), pp. 527-528. 
toute façon, dans les deux États allemands, la part des investissements se situait à un niveau assez élevé.

Par contre, en terme de PNB par habitant, celui-ci se situe à un niveau inférieur $\left(78 \%\right.$ du niveau de la RFA $^{140}$ et même à $67 \%$ pour le PNB par actif, ce qui s'explique par le fait que le taux d'emploi est plus élevé en RDA). De ce fait, maintenir le taux d'investissement comparable à celui de son homologue de l'Ouest a été une charge supérieure en $\mathrm{RDA}^{141}$. Mais le rapport reste stable alors que sur la période, la croissance du PNB par habitant a été d'environ $64 \%$ pour la RFA (69\% pour la RDA) ${ }^{142}$, donc une croissance parallèle, et même un peu meilleure pour la RDA.

A l'Ouest, une grande partie de ce financement est du ressort des entreprises que celles-ci assurent essentiellement avec l'autofinancement (jusqu'au début des années 1970), tandis qu'à l'Est celui-ci vient de l'État. Car l'une des différences majeures dans le financement de l'économie est évidemment la forme de la propriété. En RFA, les entreprises sont toutes privées, il n'y a eu aucune nationalisation, malgré l'épuration économique après la guerre (et malgré le souhait du $\operatorname{SPD}^{143}$ à cette époque), parce que les Alliés redoutaient de laisser aux mains de l'État allemand de trop grands moyens de puissance (en particulier dans l'industrie lourde). Cependant, jusqu'en $1982^{144}$, l'État conserve un certain nombre d'entreprises publiques (chemins de fer, poste, compagnie aérienne, téléphone), mais qui relèvent toutes des services publics et non du secteur de la production industrielle.

En RDA, dans un premier temps, le régime n'a pas interdit la propriété privée, en particulier dans les petites entreprises et dans l'artisanat. Ainsi, en $1950,23,5 \%$ de la propriété industrielle est encore privée. Mais très vite, des orientations idéologiques qui se durcissent conduisent à une nationalisation presque totale (les entreprises entièrement privées ne représentent plus que $12,7 \%$ du total en $1955,3,8 \%$ en 1960 et seulement $1,5 \%$ en 1969) ${ }^{145}$.

La forte croissance économique qui marque les années 1960 dans les deux pays, a permis la construction de bases économiques solides. Pourtant, le début des années 1970 révèle certaines limites à cette réussite parallèle, et montre également que les deux systèmes tendent à diverger plus fortement.

Dès 1971, des tensions monétaires internationales pèsent sur l'économie ouest-allemande et la crise pétrolière va amplifier les difficultés. Mais malgré

\footnotetext{
${ }^{140}$ Werner Obst indique un rapport variant de $75 \%$ (1961) à $77 \%$ (1972) ; OBST (note 56), tableau 3, p. 19.

${ }^{141}$ GREGORY, LEPTIN (note 1), p. 528.

${ }^{142}$ OBST (note 56).

${ }^{143}$ Celui-ci y renonce en 1959 (programme de Bad Godesberg), tout comme il renonce aussi à la planification et décide de promouvoir la cogestion.

${ }^{144}$ Date de l'arrivée au pouvoir d'Helmut Kohl qui va mettre en œuvre une politique de privatisation de ces services.

${ }^{145}$ Heinz JUNG et al., BRD-DDR. Vergleich der Gesellschaftssysteme, Cologne, 1971.
} 
le large repli de la RDA sur le monde communiste, qui en théorie aurait dû lui épargner les conséquences de la première grande crise mondiale de l'aprèsguerre, son économie en subit néanmoins les contrecoups. En effet, l'URSS augmenta le prix de ses matières premières, ce qui toucha essentiellement les pays du CAEM, et donc la RDA, très dépendante vis-à-vis de l'Union soviétique. Ainsi, la balance commerciale est pénalisée par l'augmentation de la facture énergétique qui mobilise alors un tiers de ses bénéfices du commerce avec l'URSS, le double par rapport aux années d'avant la crise ${ }^{146}$.

Ainsi, malgré des systèmes et des marchés totalement opposés, les deux Allemagne subirent chacune à leur manière le premier puis le second choc pétrolier. La RFA mit près d'une décennie à s'en remettre vraiment, mais l'économie de la RDA commença à partir de cette date son déclin inexorable.

Dès l'émergence d'un État soviétique qui à l'évidence développait une économie sur des bases très différentes des pays occidentaux, certains économistes avaient discuté une «théorie de la convergence » selon laquelle les économies capitalistes et socialistes deviennent progressivement similaires et évoluent dans la même direction. Ils l'ont développé au début des années 1960, à propos des deux Allemagne (en particulier, Walt W. Rostow, Raymond Aron et Jan Tinbergen $)^{147}$. En somme, selon les défenseurs de cette théorie, le capitalisme deviendrait moins « capitaliste » et le socialisme, moins « socialiste ». La convergence vers un « stade final optimal» ne serait qu'une question de temps ${ }^{148}$.

Ces tendances générales techniques et économiques seraient concrétisées par un même niveau de développement technologique (et en particulier, de mêmes techniques de planification, de calculs des coûts et de l'efficacité); une même structure de la production (en particulier un processus de concentration); des formes d'organisation similaires (avec des corporations dominées par des managers/technocrates tant dans les secteurs privés que publics) ; des investissements de mêmes formes et visant les mêmes objectifs ; un même niveau de croissance; les mêmes progrès techniques ; un système de revenus convergeant vers une répartition plus équitable; les mêmes standards de vie et de consommation (niveau élevé dans les deux systèmes); une éducation similaire (élévation générale du niveau); une notion de "Welfare State» (sécurité sociale assurée); une forte urbanisation et des relations commerciales extérieures développées (pas d'autarcie).

Dans le domaine des politiques économiques, on peut noter une convergence également vers une forte influence du gouvernement dans les activités économiques; des buts identiques de la politique économique (niveau

\footnotetext{
${ }^{146}$ SCHNEILIN, SCHUMACHER (note 10), p.80.

147 Jutta KNEISSL, «The Convergence Theory: The Debate in the Federal Republic of Germany », in : New German Critique, (Spring 1974), p. 16.

${ }^{148}$ Idem.
} 
de vie, plein emploi, croissance, sécurité sociale) et de mêmes formes de management (tendance à une planification centrale combinée avec la coordination des marchés et la régulation des secteurs instables) ${ }^{149}$.

Dans le domaine de la propriété des moyens de production, ce qui semble converger c'est le contrôle sur cette propriété. Dans les deux systèmes, elle a tendance à passer entre les mains des managers ou de spécialistes hautement qualifiés, qui à l'Ouest remplacent les propriétaires (pour les décisions entrepreneuriales) et dans les pays socialistes remplacent les officiels du parti. Donc dans les deux systèmes, on voit la création d'une nouvelle classe de nonpropriétaires qui assument des positions de direction sur la base de leur éducation et de leurs compétences ${ }^{150}$. Dans l'ensemble, cette approche théorique semble s'adapter à la lecture du cas des deux économies allemandes, au moins pour les années 1960 .

Cependant, certaines restrictions peuvent être apportées à cette théorie de la convergence, car elle est basée sur l'idée que la rationalité économique déterminera le développement futur, en négligeant totalement le cadre politique $^{151}$. Mais bien sûr, des systèmes économiques et sociaux à un niveau avancé de division du travail ont en commun un certain nombre de principes d'organisation. De même, des structures techniques similaires conduisent inévitablement à des systèmes proches ${ }^{152}$.

Cependant, du côté de la RDA, même si l'on admet certaines similarités d'apparence, cette théorie est totalement rejetée, pour deux raisons majeures. Tout d'abord une insurmontable différence de la base socio-économique (en particulier dans les relations de propriété) et un contrôle et une gestion économique planifiée vers des buts socialistes qui s'opposent à la production non coordonnée et semée de crises des pays capitalistes. Selon les théoriciens de la RDA, cette théorie serait en fait une des idées-clés de l'anticommunisme du temps (et aussi la vision d'un «ordre optimal $»)^{153}$. On fait remarquer de plus que cette théorie est en accord avec la nouvelle Ostpolitik du gouvernement ouest-allemand. Elle vise à terme l'idée d'une domination et d'une acceptation des méthodes économiques capitalistes. Donc cette théorie suit les buts de l'impérialisme capitaliste, elle correspond à une stratégie globale et elle sert de fondation et de justification à la nouvelle Ostpolitik. Le gouvernement social-démocrate s'inscrirait parfaitement dans ce concept. Donc même si les traités entre la RDA et la RFA (1972) sont le résultat positif de la politique est-allemande de détente, en même temps, sur la base de ce

\footnotetext{
${ }^{149}$ KNEISSL (note 147), p. 18.

${ }^{150}$ KNEISSL (note 147), p. 22.

${ }^{151}$ Selon Wolfgang BEHR (note 17), p. 123, les deux systèmes économiques ont des éléments de similarité, mais pour autant, on ne peut en conclure à des éléments de convergence.

${ }^{152}$ KNEISSL (note 147), pp. 22-23.

${ }^{153}$ Idem.
} 
succès et de la coopération croissante avec l'Ouest, les représentants du parti estiment cependant que le combat idéologique doit être, dans le même temps, intensifié $^{154}$.

Si la convergence n'est donc pas acquise, mais pas encore contestée par les faits au début des années 1970, il n'en reste pas moins que sur cette période d'une quinzaine d'années il y a deux économies qui, bien que fonctionnant sur des bases très différentes, n'en sont pas moins des modèles qui affichent certaines réussites, dans une relation de forte concurrence qui a été probablement très stimulante pour les deux États frères (ennemis).

${ }^{154}$ KNEISSL (note 147), pp. 24-25. 